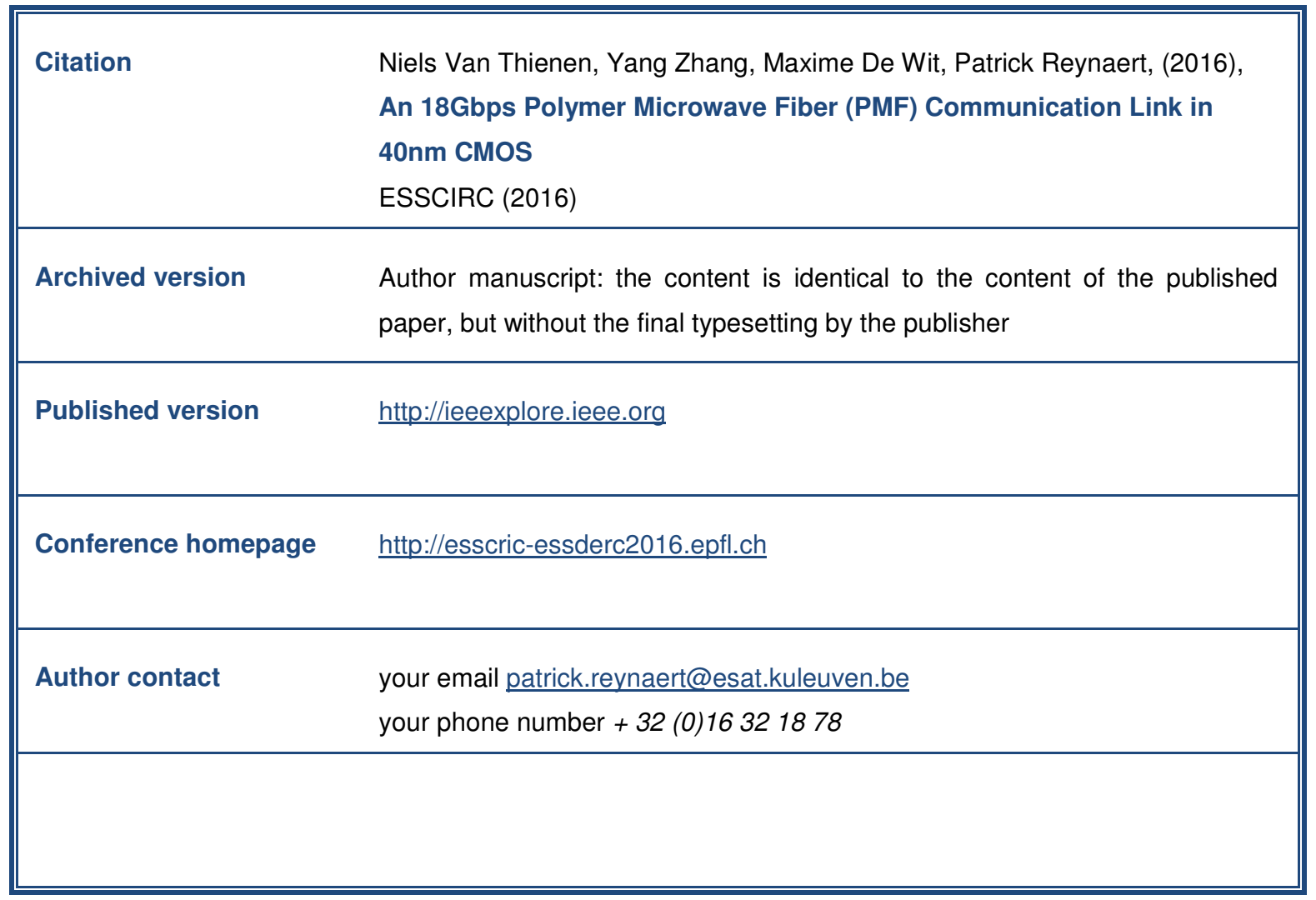

(article begins on next page) 


\title{
An 18Gbps Polymer Microwave Fiber (PMF) Communication Link in 40nm CMOS
}

\author{
Niels Van Thienen, Yang Zhang, Maxime De Wit and Patrick Reynaert \\ KU Leuven ESAT-MICAS, Kasteelpark Arenberg 10, 3001 Leuven, Belgium \\ patrick.reynaert@esat.kuleuven.be
}

\begin{abstract}
A high-speed polymer microwave fiber (PMF) communication link is implemented in a $40 \mathrm{~nm}$ bulk CMOS technology, by making use of two-tone CP-FSK modulation and a carrier frequency of $120 \mathrm{GHz}$. The presented transceiver and enhanced coupler, with a loss of only $3.4 \mathrm{~dB}$, allows communication over a distance of $15 \mathrm{~m}$ at $1.5 \mathrm{Gbps}$. Data rates up to 17.7Gbps were achieved over a link distance of $1 \mathrm{~m}$ with a BER $<10^{-12}$. The maximum product of data rate and distance is 60.8Gbps.m over a distance of $8 \mathrm{~m}$, resulting in an energy efficiency of only $1.2 \mathrm{pJ} / \mathrm{bit} / \mathrm{m}$. The transmitter has an output power level of $-1.9 \mathrm{dBm}$ at $120 \mathrm{GHz}$ and a DC power consumption of $11.1 \mathrm{~mW}$, the receiver consumes $59.6 \mathrm{~mW}$. The transmitter and receiver occupy an active area of respectively $0.025 \mathrm{~mm}^{2}$ and $0.43 \mathrm{~mm}^{2}$ in $40 \mathrm{~nm}$ CMOS.
\end{abstract}

Keywords - Plastic waveguide, polymer microwave fiber, tapered slot coupler, multi-gigabit, CMOS, mm-Wave.

\section{INTRODUCTION}

The continuous CMOS technology scaling not only allows for high-performance baseband communication systems, but also increases $f_{\max }$, thereby improving the mm-Wave performance of CMOS integrated circuits for data transmission. A $\mathrm{mm}$-Wave wireless communication system benefits from the inherent larger absolute bandwidth available at these high frequencies. However, the limiting factor with $\mathrm{mm}$-Wave wireless communications is the short distance due to the increased freespace path loss.

The proposed directive polymer channel in [1]-[6] provides a wired solution to this problem where mm-Wave carriers stay confined within a plastic waveguide. A hollow Teflon tube with an outer and inner diameter of respectively 2 and $1 \mathrm{~mm}$, can act as a directive channel, with a loss of $2.5 \mathrm{~dB} / \mathrm{m}$ at $120 \mathrm{GHz}$ compared to more than $97.5 \mathrm{~dB}$ for a $15 \mathrm{~m}$ link in free space [1]. This makes PMF technology suitable for a variety of applications, such as automotive, industrial, aviation and consumer electronics, where a light-weight, low-cost and high-speed operation is desired in the range of 1 to $15 \mathrm{~m}$.

The coupling loss between chip and plastic waveguide becomes an important factor. It is challenging to launch an onchip $120 \mathrm{GHz}$ modulated signal into the plastic waveguide with minimal losses. On-chip antennas as proposed in [1], despite being very compact, have major inherent drawbacks caused by the silicon substrate, resulting in sub-optimal constructive reflection and therefore a low radiation efficiency. In this work a dedicated tapered slot coupler is proposed as a solution to increase the link distance. In contrast to the on-chip collinear

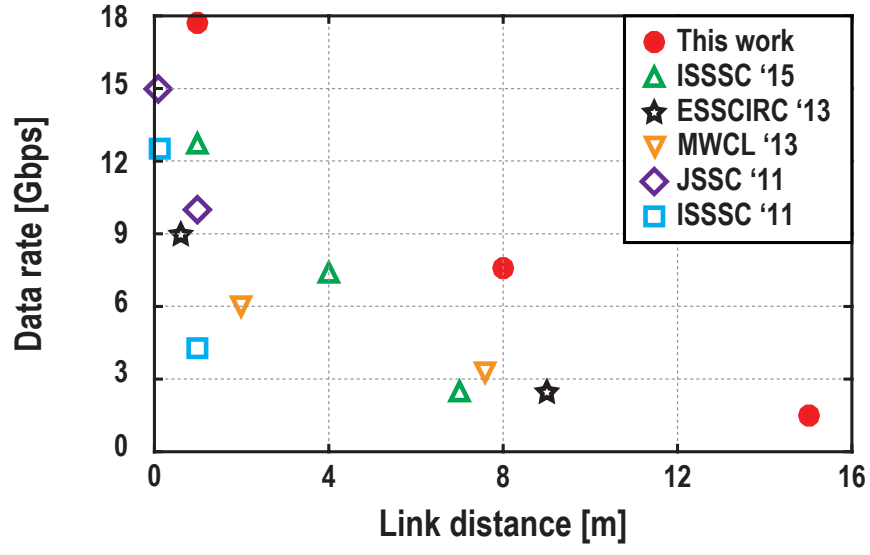

Fig. 1. Comparison of this work to previously published polymer microwave fiber communication links.

broadside dipole antenna proposed in [1], this work presents a transmitter and a receiver which are optimized for an external $120 \mathrm{GHz}$ tapered slot coupler, leading to a $16 \mathrm{~dB}$ improvement of the entire communication link. The improvement of the coupling efficiency made it feasible to design a working $15 \mathrm{~m}$ link.

This paper presents a $120 \mathrm{GHz}$ PMF communication link using 40nm CMOS chips. A tapered slot coupler, with a measured loss of only $3.4 \mathrm{~dB}$ and a bandwidth of more than $40 \mathrm{GHz}$, results in communication through a15m long polymer fiber at a data rate of $1.5 \mathrm{Gbps}$. Compared to prior published PMF communication links, this transceiver achieves the highest reported data rate of $17.7 \mathrm{Gbps}$ over a distance of $1 \mathrm{~m}$, as seen in fig. 1 , and is able to reach a data rate of $7.6 \mathrm{Gbps}$ over $8 \mathrm{~m}$, resulting in an energy efficiency of only $1.2 \mathrm{pJ} / \mathrm{bit} / \mathrm{m}$. Design optimizations have been performed in the transistor layout, link budget, flip-chip packaging and the lowloss tapered slot coupler.

\section{TOPOLOGY AND CIRCUIT IMPLEMENTATION}

An overview of the complete PMF communication link is illustrated in fig. 2. An off-chip clock triggers the on-chip data generator, which modulates a $120 \mathrm{GHz}$ carrier according to a two-tone Continuous Phase Frequency Shift Keying (CPFSK) modulation technique. A wide-band coupler launches the modulated EM-wave in the teflon waveguide. The same 


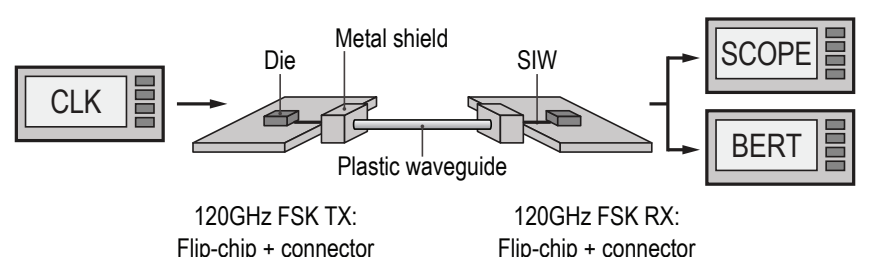

Fig. 2. System overview and measurement setup for the $120 \mathrm{GHz}$ PMF communication link.

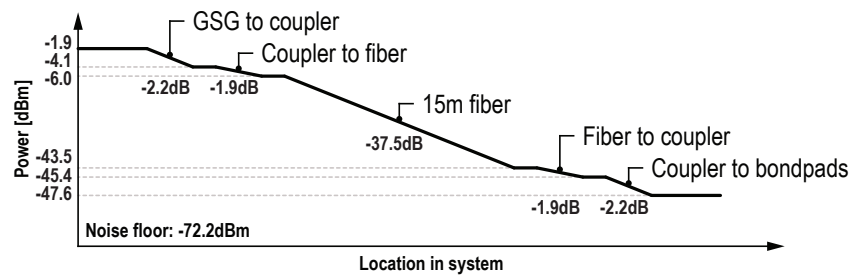

Fig. 3. Link budget analysis for a 15m PMF communication link.

coupler is used at the receiver side, where the incoming signal is demodulated, after which a post-amplifier drives the $50 \Omega$ load of the measurement equipment. A detailed link budget analysis is given in fig. 3, including the measured values of the low-loss couplers. The transmitted power level at the GSG output pads amounts $-1.9 \mathrm{dBm}$. The two couplers introduce a loss of $3.4 \mathrm{~dB}$ each, the $15 \mathrm{~m}$ long plastic waveguide a loss of $37.5 \mathrm{~dB}$. This results in an input SNR of $24.6 \mathrm{~dB}$ at the receiver side, with a thermal noise floor of $-72.2 \mathrm{dBm}$ over a bandwidth of $10 \mathrm{GHz}$.

CP-FSK modulation was chosen as it has a constant envelope waveform, thus allowing low-power and highly-efficient non-linear amplifiers throughout the entire communication link, improving the energy efficiency. Non-coherent detection is an important advantage of this modulation technique, resulting in a less complex receiver topology compared to, for example, a phase-shift keying (PSK) demodulator. Also, CP-FSK does not require a variable gain amplifier, because the carrier amplitude holds no information. Finally, CP-FSK is resistant against LO pulling [7], which appears when an oscillator, a power amplifier and an antenna are integrated on the same chip.

\section{A. Transmitter}

The transmitter, equipped with both an on-chip PRBS9 generator and an external data input, modulates an on-chip generated $120 \mathrm{GHz}$ carrier as described in fig. 4 . The $120 \mathrm{GHz}$ VCO is implemented with an LC-tank, driven by a crosscoupled differential pair. In parallel with the LC-tank, a switched capacitor handles the CP-FSK modulation. A selector applies one of both input signals, a PRBS9 sequence or external data, to the switched capacitor. The resonance frequency of the LC-tank [1] changes, resulting in a twotone CP-FSK modulation with approximately $4 \mathrm{GHz}$ spacing in between. Two additional switched capacitors can be used to change the fundamental frequency in steps of $2 \mathrm{GHz}$.

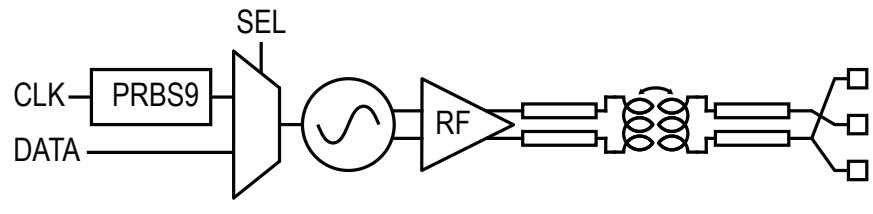

Fig. 4. Block diagram of the $120 \mathrm{GHz}$ CP-FSK transmitter. Equipped with an on-chip PRBS9 generator and an external data input.

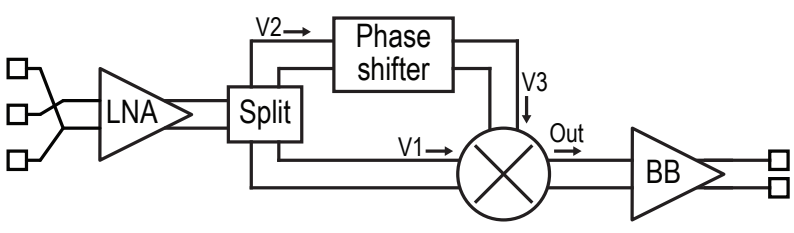

Fig. 5. Block diagram of the $120 \mathrm{GHz}$ CP-FSK receiver, consisting of an LNA, a splitter, a Gilbert-cell mixer and a baseband amplifier to drive a $50 \Omega$ off-chip load.

An off-chip clock triggers the PRBS9 generator, which generates bit sequences at twice the clock frequency, resulting in transmitted data rates up to $20 \mathrm{Gbps}$. An amplifier buffers the modulated output of the VCO and a matching network handles the transformation to the output pads which see a $50 \Omega$ load through the flip-chip gold bumps. A transmitted output power of $-6 \mathrm{dBm}$ at $120 \mathrm{GHz}$ was measured, including the matching losses towards the plastic waveguide. The transmitter consumes only $11.1 \mathrm{~mW}$ from $0.9 \mathrm{~V}$. The low power consumption is a result of integrating both carrier generation and modulation in one building block using CP-FSK modulation.

\section{B. Receiver}

Fig. 5 describes the block diagram of the CP-FSK receiver. The incoming signal from the GSG input pads is amplified by a 5-stage low-noise amplifier (LNA), as shown in fig. 6. The LNA has a total gain of $26.8 \mathrm{~dB}$ and a simulated noise figure of $6.6 \mathrm{~dB}$. Each stage of the LNA consists of a neutralized pair to ensure differential mode stability. Also, attention was paid on the common mode stability during the design, this was done by adding resistors to the transformer center taps of the biasing network [8]. The last stage of the LNA drives another neutralized pair of which the transformer-based output matching network splits the signal in V1 and V2 as illustrated on the block diagram in fig. 5. By introducing a frequency dependent phase shift in one of these paths, V2 becomes V3. The phase shifter translates the frequency modulated signal into a phase modulated signal, which can be detected by direct downconversion, resulting in an amplitude baseband signal, which is amplified by a two-stage post-amplifier. Finally a measurement buffer drives the $50 \Omega$ measurement equipment. The receiver has a DC power consumption of $59.6 \mathrm{~mW}$, including the LNA, phase shifter, mixer and the two-stage postamplifier. 


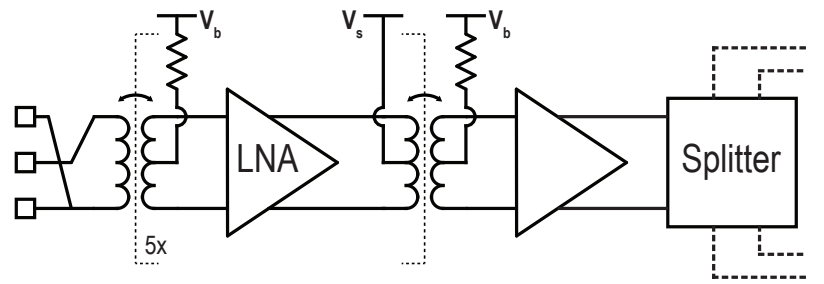

Fig. 6. Schematic of the 5-stage LNA with a resistive bias network connected to the center taps of the top-metal transformers to ensure common mode stability.

\section{LOW-LOSS TAPERED SLOT COUPLER}

In order to improve the coupling efficiency from the chip towards the plastic waveguide, either the waveguide ending has to be modified [4] to increase the ability to capture high frequency signals, or an antenna array is needed to enhance the directivity [1]. The improved solution proposed in this work is a tapered slot coupler on a low-loss Megtron 6 substrate, as illustrated in fig. 7. Flip-chip packaging not only provides a robust interconnect from the bondpads to the substrate integrated waveguide (SIW), but also has lower losses and parasitics than a bondwired package.

As shown in fig. 7, the matching network is implemented with an SIW, realizing an impedance transformation from $50 \Omega$ to $59 \Omega$. In this work, the size of the bondpads for the gold bumps is $60 \mu \mathrm{m}$ by $60 \mu \mathrm{m}$ with a $120 \mu \mathrm{m}$ pitch. The Megtron 6 substrate is $300 \mu \mathrm{m}$ thick with a relative permittivity of 3.6. The tapered slot antenna realizes a mode transition from TE10 mode in the SIW towards an HE11 mode in the plastic waveguide. The coupler and plastic waveguide ending are shielded by a metal enclosure to achieve good isolation from the environment and to realize plastic waveguide selfalignment, therefore no extra fastening is needed. A loss of only $1.9 \mathrm{~dB}$, including the substrate loss, was measured at a frequency of $120 \mathrm{GHz}$. The entire wideband connector structure, including gold bumps, bondpads and substrate loss, has a measured loss of only $3.4 \mathrm{~dB}$ as shown in fig. 7 . Compared to the on-chip twisted dipole antenna in [1], the coupling loss is improved with $8 \mathrm{~dB}$, resulting in a $16 \mathrm{~dB}$ higher margin for the entire link without consuming more DC power. The bandwidth of the tapered slot coupler is more than $40 \mathrm{GHz}$.

\section{Measurement Results}

The measurement setup for the entire PMF communication link is illustrated in fig. 2. Three different channel lengths of $1 \mathrm{~m}, 8 \mathrm{~m}$ and $15 \mathrm{~m}$ are used during the measurements. The resulting eye diagrams for different data rates and distances are shown in fig. 8. Note that the eye opening is still very clear, despite the high data rate of $17.7 \mathrm{Gbps}$ or the $15 \mathrm{~m}$ link distance. The eye diagrams are captured with a Keysight DSO$\mathrm{Z}$ 634A 63GHz 160Gs oscilloscope, which is connected to the analog baseband output.

Fig. 8 shows the maximum achieved data rates of respectively $1.5 \mathrm{Gbps}, 7.6 \mathrm{Gbps}$ and $17.7 \mathrm{Gbps}$ for the three different channel lengths and a bathtub curve for the $8 \mathrm{~m}$ link. The bit
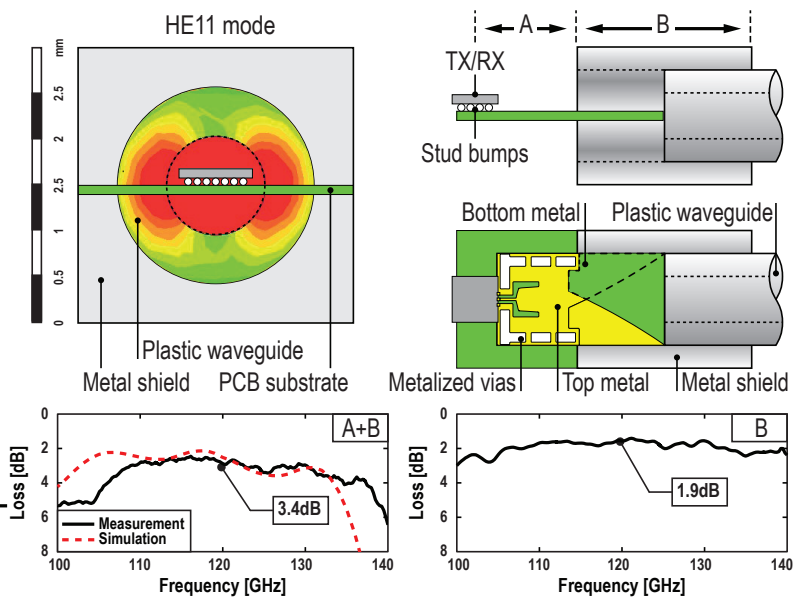

Fig. 7. Tapered slot coupler implementation on a low-loss Megtron 6 substrate with simulated and measured coupling loss from respectively substrate to fiber and bondpads to fiber. At $120 \mathrm{GHz}$ the entire connector has a measured loss of only $3.4 \mathrm{~dB}$.
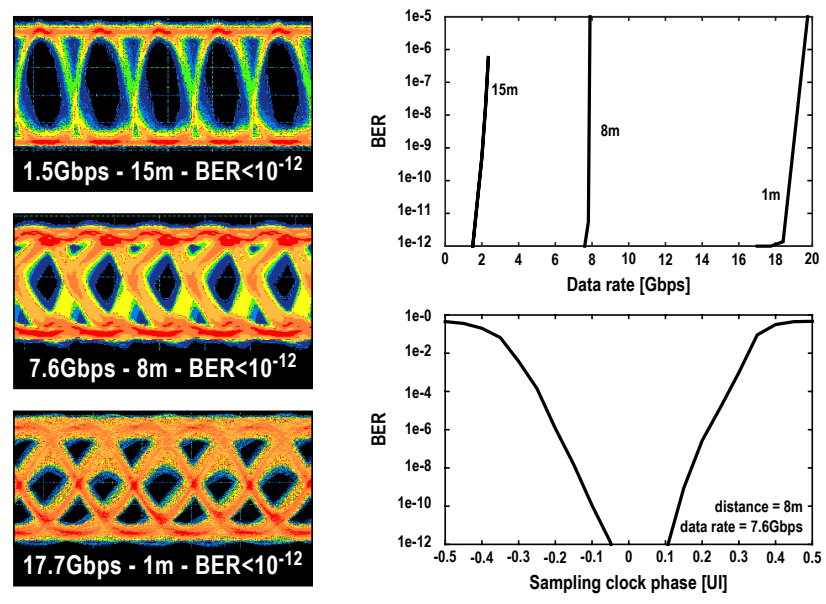

Fig. 8. Measured eye diagrams, BER curves and a bathtub curve of the baseband output at different data rates and distances with a BER $<10^{-12}$. No DFE or error correction was applied at the RX side.

error rates are measured with a Keysight 81250 ParBERT up to data rates of $13.5 \mathrm{Gbps}$. For data rates up to $32 \mathrm{Gbps}$, a Keysight N4960 BERT is used. For all BER measurements, a PRBS length of $2^{-9}-1$ is used.

Table I compares the results of this work to previously published communication links. Due to the improved coupling efficiency, this work achieves the highest reported communication distance and data rate compared to other prior published PMF communication links. A maximum distance of $15 \mathrm{~m}$ was measured at a data rate of $1.5 \mathrm{Gbps}$. Data rates up to $17.7 \mathrm{Gbps}$ were achieved over a distance of $1 \mathrm{~m}$. A maximum product of data rate and distance of $60.8 \mathrm{Gbps} . \mathrm{m}$ was measured over a distance of $8 \mathrm{~m}$, resulting in an energy efficiency of $1.2 \mathrm{pJ} / \mathrm{bit} / \mathrm{m}$. This without any decision feedback equalization (DFE) or error correction employed in any of the measurements, while still maintaining a BER below $10^{-12}$. 
TABLE I

Performance Summary and Comparison of Previously Published State-of-the-Art PMF Communication Links.

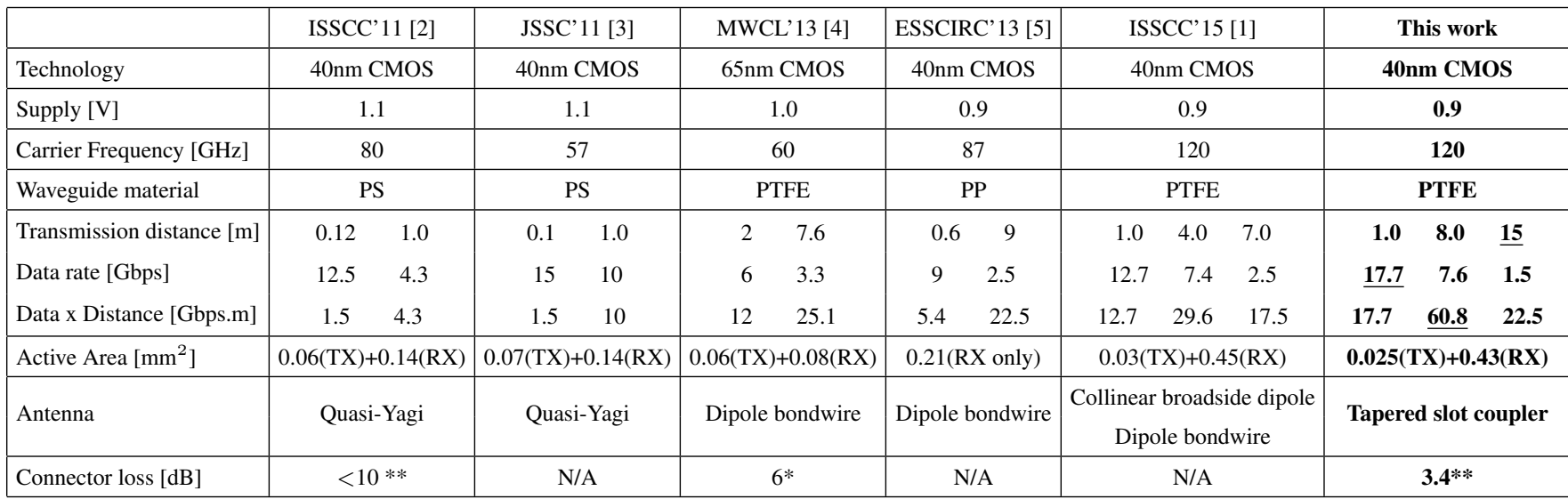

* Simulated coupling loss

*** Measured coupling loss from chip to PMF
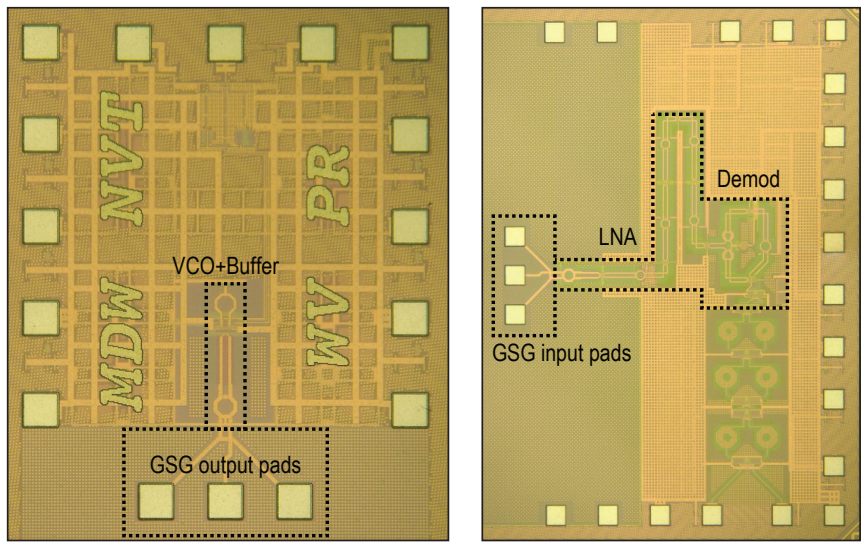

Fig. 9. Die micrographs of TX (left) and RX (right) in 40nm CMOS. TX and RX occupy an active area of respectively $0.025 \mathrm{~mm}^{2}$ and $0.43 \mathrm{~mm}^{2}$.

The output stage of the transmitter has a measured power level of $-1.9 \mathrm{dBm}$. The receiver has a DC power consumption of $59.6 \mathrm{~mW}$, the transmitter consumes only $11.1 \mathrm{~mW}$ when transmitting $7.6 \mathrm{Gbps}$ over $8 \mathrm{~m}$. The entire communication link consumes $70.7 \mathrm{~mW}$. The transmitter and receiver occupy an active area of respectively $0.025 \mathrm{~mm}^{2}$ and $0.43 \mathrm{~mm}^{2}$. The transmitter and receiver chips are implemented in a $40 \mathrm{~nm}$ CMOS technology and the die micrographs are shown in fig. 9.

\section{CONCLUSiON}

A multi-gigabit PMF communication link was proposed and implemented in 40nm CMOS. Channel measurements, system and circuit level implementations and coupling structures are presented in this work. The performance of the transmitter and receiver were measured and reported. A maximum distance of $15 \mathrm{~m}$ was measured at a data rate of $1.5 \mathrm{Gbps}$, data rates up to $17.7 \mathrm{Gbps}$ were achieved over a distance of $1 \mathrm{~m}$ and an energy efficiency of $1.2 \mathrm{pJ} / \mathrm{bit} / \mathrm{m}$ was achieved. The transmitter with an active area of $0.025 \mathrm{~mm}^{2}$ achieves a measured output power level of $-1.9 \mathrm{dBm}$. The tapered slot coupler has a minimum loss of only $3.4 \mathrm{~dB}$, which is an improvement of $8 \mathrm{~dB}$ compared to the on-chip collinear broadside dipole antenna in [1], and a more than $40 \mathrm{GHz}$ bandwidth. Table I provides a detailed performance summary and compares this work to previously published state-of-the-art PMF links, showing the fastest and longest PMF communication link reported today.

\section{REFERENCES}

[1] W. Volkaerts, N. Van Thienen, and P. Reynaert, "An FSK plastic waveguide communication link in 40nm CMOS," in Solid-State Circuits Conference Digest of Technical Papers (ISSCC), 2015 IEEE International, pp. 178-179, Feb 2015.

[2] S. Fukuda, Y. Hino, S. Ohashi, T. Takeda, S. Shinke, M. Uno, K. Komori, Y. Akiyama, K. Kawasaki, and A. Hajimiri, "A $12.5+12.5 \mathrm{~Gb} / \mathrm{s}$ full-duplex plastic waveguide interconnect," in Solid-State Circuits Conference Digest of Technical Papers (ISSCC), 2011 IEEE International, pp. 150-152, Feb 2011.

[3] S. Fukuda, Y. Hino, S. Ohashi, T. Takeda, H. Yamagishi, S. Shinke, K. Komori, M. Uno, Y. Akiyama, K. Kawasaki, and A. Hajimiri, "A 12.5+12.5 Gb/s Full-Duplex Plastic Waveguide Interconnect," Solid-State Circuits, IEEE Journal of, vol. 46, no. 12, pp. 3113-3125, Dec 2011.

[4] Y. Kim, L. Nan, J. Cong, and M.-C. Chang, "High-Speed mm-Wave DataLink Based on Hollow Plastic Cable and CMOS Transceiver," Microwave and Wireless Components Letters, IEEE, vol. 23, no. 12, pp. 674-676, Dec 2013.

[5] M. Tytgat and P. Reynaert, "A plastic waveguide receiver in 40nm CMOS with on-chip bondwire antenna," in ESSCIRC (ESSCIRC), 2013 Proceedings of the, pp. 335-338, Sept 2013.

[6] M. Tytgat, N. Van Thienen, and P. Reynaert, "A 90-GHz receiver in 40nm CMOS for plastic waveguide links," Analog Integrated Circuits and Signal Processing, vol. 83, no. 1, pp. 55-64.

[7] W. Volkaerts, M. Steyaert, and P. Reynaert, "A $120 \mathrm{GHz}$ QVCO with $16.2 \mathrm{GHz}$ tuning range resistent against VCO pulling in $45 \mathrm{~nm}$ CMOS," Analog Integrated Circuits and Signal Processing, vol. 82, no. 2, pp. 359-368, 2015.

[8] N. Deferm and P. Reynaert, "Differential and common mode stability analysis of differential mm-wave CMOS amplifiers with capacitive neutralization," Analog Integrated Circuits and Signal Processing, vol. 80, no. 1, pp. 1-12, 2014. 